\title{
Evaluation of tracheal stenosis: comparison between computed tomography virtual tracheobronchoscopy with multiplanar reformatting, flexible tracheofiberoscopy and intra-operative findings
}

\author{
Kamal Morshed • Agnieszka Trojanowska • \\ Marcin Szymański • Piotr Trojanowski • \\ Anna Szymańska · Agata Smoleń · Andrzej Drop \\ Received: 13 May 2010/Accepted: 1 September 2010/Published online: 17 September 2010 \\ (C) The Author(s) 2010. This article is published with open access at Springerlink.com
}

\begin{abstract}
The aim of the study was to evaluate and compare various helical CT display modes [virtual endoscopy (VE)] and multiplanar reformations (MPR), conventional flexible tracheobronchoscopy (FT) and intra-operative (IO) findings in patients with tracheal stenosis and to analyze the advantage of MPR and VE in diagnosis and treatment planning and in postoperative follow-up. Thirty-seven patients with tracheal stenosis underwent standard neck and chest CT followed by MPR and VE. Results were correlated with the results of FT and IO findings. Thirty-three of the 37 stenoses were correctly graded and measured adequately using VE. Complete correlation among CT, fiberoptic tracheoscopy, and surgery of stenosis grading, stenosis length and length of planned resection segment of the trachea was noted between 33 of 37 patients with tracheal stenosis. Correlation between $\mathrm{VE}$ and $\mathrm{IO}$ was noted in 35 of 37 patients and between FT and VE was noted in 33 of 37 patients with
\end{abstract}

K. Morshed ( $₫)$ · M. Szymański · P. Trojanowski

Department of Otolaryngology Head and Neck Surgery,

Medical University of Lublin, Jaczewskiego 8 str.,

20-090 Lublin, Poland

e-mail: kamal1@op.pl

A. Trojanowska $\cdot$ A. Drop

Department of Diagnostic Radiology,

Medical University of Lublin, Jaczewskiego 8 str.,

20-090 Lublin, Poland

\section{A. Szymańska}

Department of Interventional Radiology and Neuroradiology,

Medical University of Lublin, Jaczewskiego 8 str.,

20-090 Lublin, Poland

\section{A. Smoleń}

Department of Mathematics and Biostatistics,

Medical University of Lublin, Ul Jaczewskiego 8 str.,

20-090 Lublin, Poland tracheal stenosis. The sensitivity of VE was $94-97 \%$, specificity was $100 \%$ with comparison to IO findings. The sensitivity and accuracy of MPR was 86-89\% and specificity was $100 \%$ with comparison to FT findings. The results of the study indicate that VE is an excellent, consistent, and objective technique. VE with MPR is very useful in diagnostic evaluation and treatment planning in patients with tracheal stenosis.

Keywords Tracheal stenosis - Virtual bronchoendoscopy $\cdot$ Spiral CT $\cdot$ Multiplanar reformations

\section{Introduction}

Tracheal stenosis in adults most commonly results from intubation trauma, tracheotomy, and after blunt trauma of the neck. Other causes include benign and malignant neoplasms, inflammatory disease, and systemic autoimmune diseases [3, 25]. Management policies depend on the accurate mapping of the lesion including, severity, vertical extent, the status of the peritracheal tissue and vocal cord function. Management plans depend on the accurate mapping of the lesion.

The standard diagnostic procedures used in stenotic lesions of the central airway include computed tomography (CT) and flexible tracheobronchoscopy (FT) [6, 7, 14]. Although these techniques are highly precise and reliable, they both suffer some technical limitations that could lead to inaccurate characterization of the airway disease [6]. The possibility of obtaining multiplanar reformatting (MPR) and three-dimensional (3D) reconstructions has enabled detailed demonstration of focal airway stenosis and the localization of lesions in endotracheal, peritracheal or submucosal sites [18]. Furthermore, spiral acquisition introduced virtual endoscopy (VE), a reconstruction 
technique that exploits the natural contrast between endoluminal air and surrounding tissue [11, 12], allowing "navigation" through the tracheobronchial tree with the same endoluminal perspective as an endoscopy [18, 19, 24]. The spiral CT is a non-invasive well-tolerated procedure by all patients. On the other hand the flexible tracheobronchoscopy is used for the detection and diagnosis of tracheobronchial stenosis because it permits direct visualization of the airway lumen. However, it is an invasive procedure that can cause discomfort for the patient and it often requires hospitalization and morbidity, which is also quite low. It is also difficult to evaluate airway caliber and morphology beyond a high-grade stenosis [13].

The aim of the study was to evaluate and compare various helical CT display modes [virtual endoscopy (VE)] and multiplanar reformations (MPR), conventional flexible tracheobronchoscopy (FT) and intra-operative (IO) findings in patients with tracheal stenosis and to analyze the advantage of MPR and VE in diagnosis and treatment planning and in postoperative follow-up.

\section{Materials and methods}

\section{Patients}

Between May 2001 and December 2008, 37 patients with tracheal stenosis were examined: 29 males and 8 females, aged between 16 and 75 years. The post-intubation, posttracheotomy, or prolonged tracheotomy was the most frequent etiology. This group of consecutive patients underwent CT examination, FT and were operated by one surgeon, the same for each patient in the ENT Head and Neck Surgery Department. Patients underwent neck and chest CT followed by MPR and VE in Radiology Department. The study consisted of patients who had tracheal stenosis suitable for surgical treatment (laser treatment, tracheal resection with end-to-end anastomosis, or T-tube stent) and graded as grade 3 or 4 as inclusion criteria. The tracheal stenosis not suitable for surgical treatment was indicated as exclusion criteria.

\section{Neck and chest CT}

Images were acquired with a multislice Light Speed VCT 64 scanner (General Electric, GEMS, Milwaukee, USA) with the following technical parameters: $8 \times 1.25 \mathrm{~mm}$ collimation, 0.6-mm overlap, 0.5 -s rotation time, $120 \mathrm{~mA}$ and $120 \mathrm{kVp}$. Patients were positioned supine on the CT table, and images were acquired in the craniocaudal direction from the soft palate to the right diaphragmatic dome. Acquisition time was roughly $6 \mathrm{~s}$ to allow completion of the examination during a single breath-hold.
CT image reconstruction

Source images were transferred to a computer workstation (Advantage Windows 4.0; GEMS, Milwaukee, WI, USA). Standard reconstruction software (Navigator tool, version 2.03; GEMS) was used for virtual endoscopic images. An upper threshold of $-500 \mathrm{H}$ was applied to reconstruct the airways (multicolor mode). Each endoscopic image simulated a coned-down view, with a cone angle adjusted to $45^{\circ}$. Navigation through the trachea was performed in the fly-through mode beginning in the upper end of trachea and continuing downwards (Fig. 2). In addition, standard reformatted coronal and sagittal images of the trachea were obtained (Figs. 1, 3, 4).

\section{Flexible tracheobronchoscopy (FT)}

FT was performed by an experienced ENT surgeon using a videotracheobronchoscope (Olympus Optical, Tokyo, Japan); patients underwent standard local anesthesia before the procedure (Fig. 5). The mean interval between flexible tracheoscopy and virtual tracheoscopy was 12 days (range 7-21 days). To provide a standard of reference, an experienced ENT surgeon reviewed the FT findings first and reported without knowledge of the CT findings.

The degree of tracheal stenosis was classified according to Cotton: grade 1 , luminal narrowing $<50 \%$; grade 2 , luminal narrowing 51-70\%; grade 3, luminal narrowing $71-99 \%$ and grade 4 , luminal narrowing $100 \%$. The grading system was

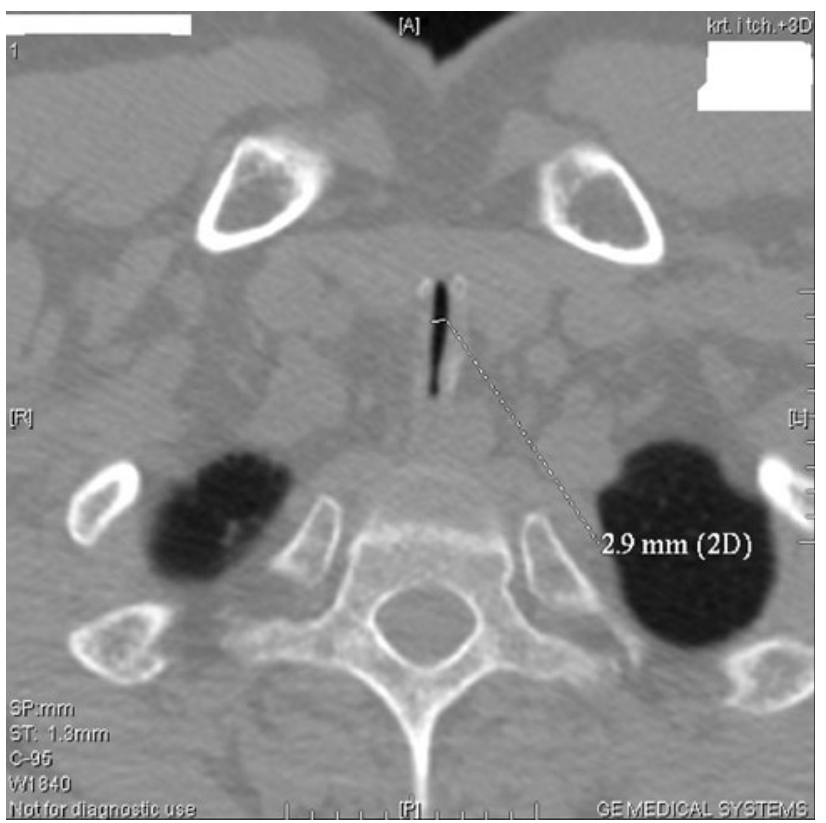

Fig. 1 Axial image with measurement of the width (diameter) of trachea 


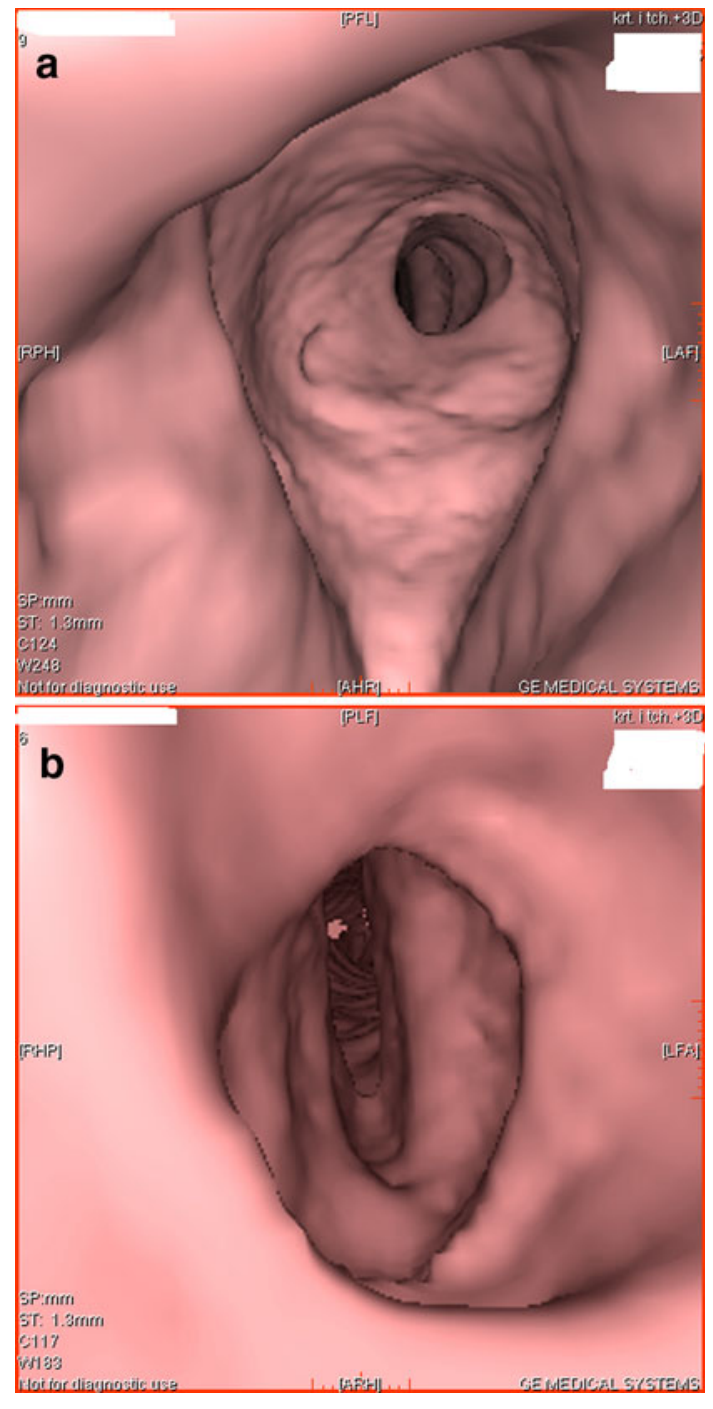

Fig. 2 Virtual endoscopy showing concentric narrowing of the airway. a View of the proximal part of stenosis, $\mathbf{b}$ view of the distal part of stenosis

used for FT, VE, MPR reconstruction (Fig. 3c, d), and intraoperative findings (Fig. 6).

The diameters, lengths, planned, and resected stenoses were measured as following:

1. Intra-operative findings (length and diameter) were measured by ruler $(\mathrm{cm})$.

2. With flexible tracheobronchoscope, the length was measured by ruler and the diameter was compared to the diameter of the flexible tracheobronchoscope.

3. In intra-operative in patients cured by laser, the measurement of diameter was done by a Laser Protector with three sizes: large $(7 \mathrm{~mm})$, medium $(5 \mathrm{~mm})$, and small $(3 \mathrm{~mm})$ (Richard Wolf Industries, Germany). Furthermore, the measurement of stenosis length was done by a ruler.

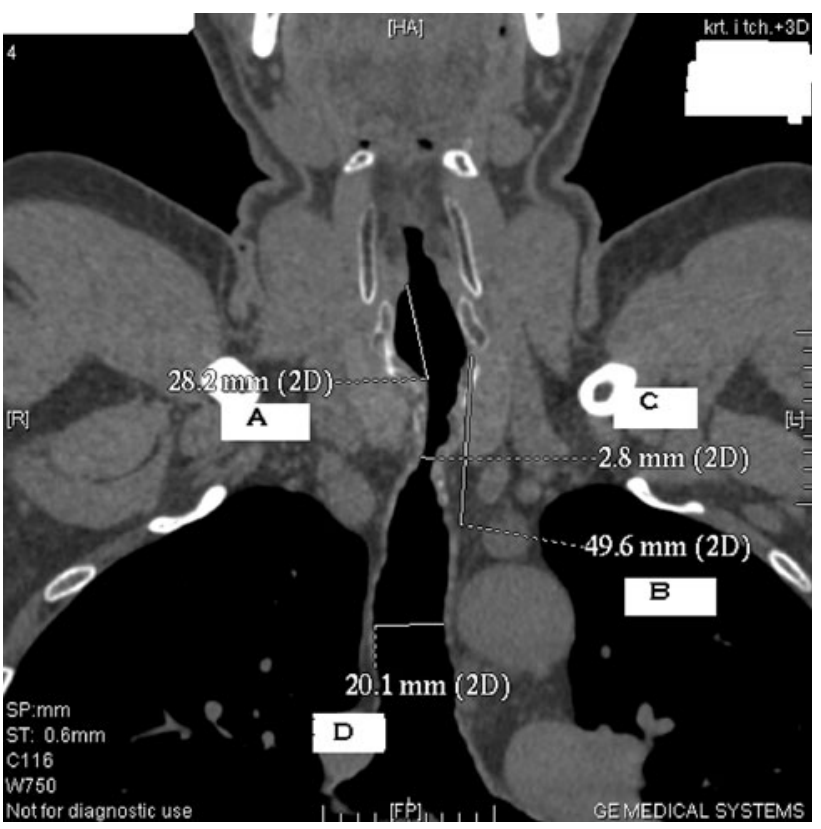

Fig. 3 Coronal image with measurements of stenosis. $A$ length from the vocal cord, $B$ length of planned segmental resection of trachea, $C$ diameter of tracheal stenosis, $D$ diameter of normal trachea

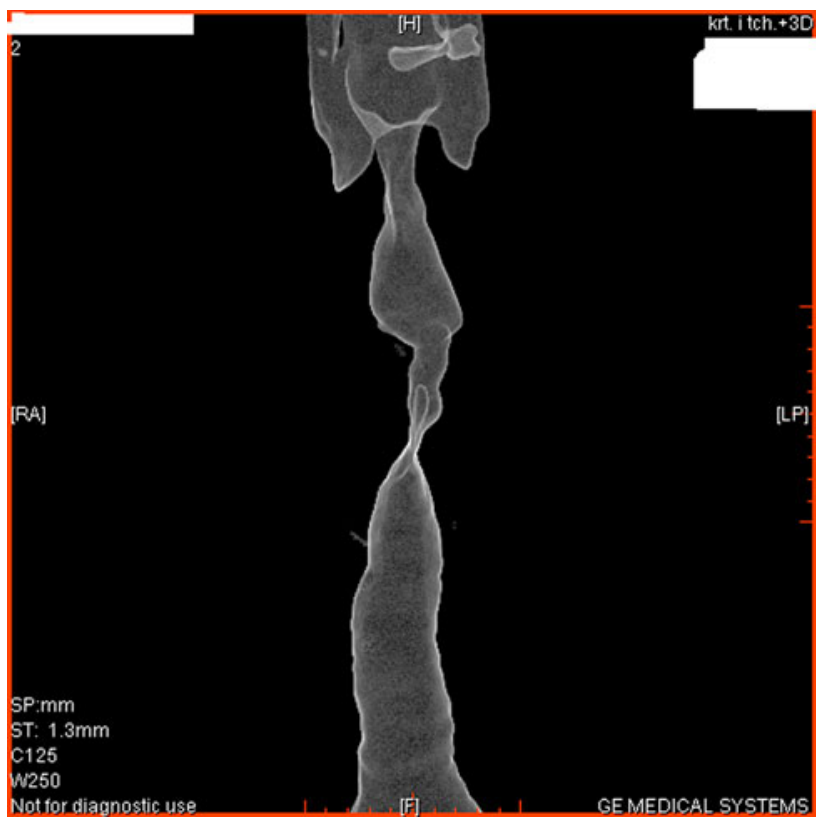

Fig. 4 3D shaded surface display

4. With t-tube stent the measurement was done during the replacement of the t-tube stent with a ruler.

5. The VE and MPR findings was measured with the program ruler.

The indication for the treatment was based on the flexible tracheobronchoscopy (FT) and radiology findings examination. The use of jet ventilation is an alternative method to the use of orotracheal tube intubation. In our 


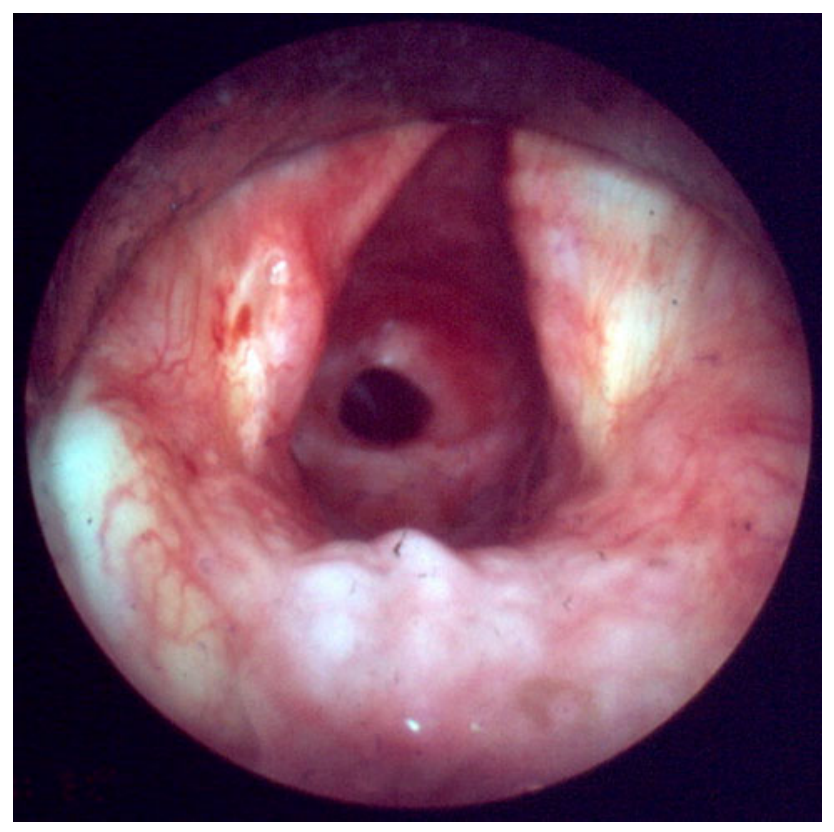

Fig. 5 Flexible tracheoscopy of tracheal stenosis

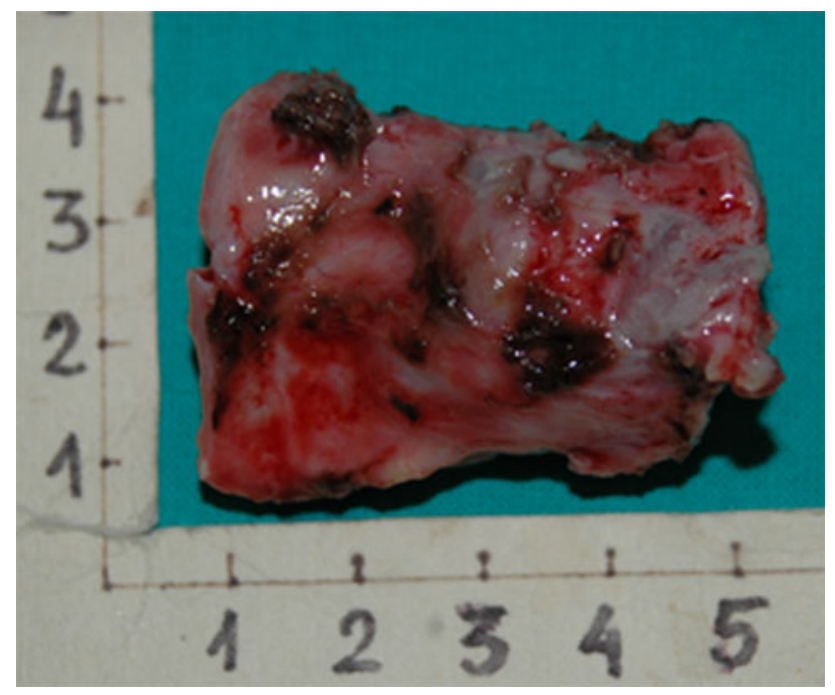

Fig. 6 Image of resected segment of trachea

patients jet ventilation was applied at the beginning of the surgery. The surgical findings served as a gold standard and the results were correlated with the FT, VE, and MPR reconstruction.

\section{Statistical analysis}

A statistical analysis was performed to evaluate the correlation between FT, VE, MPR, and intra-operative findings, with the use of $\chi^{2}$ test McNemara. $P$ value $<0.05$ was considered as statistically significant.

The study was approved by the local ethics committee.

\section{Results}

The length of stenoses ranged between 0.2 and $3.5 \mathrm{~cm}$. The distance from vocal cord to the stenotic site ranged between 2.5 and $5 \mathrm{~cm}$. The length of resected segment of the trachea ranged between 2.5 and $4.5 \mathrm{~cm}$. Thus findings were defined intra-operative. The stenosis of the trachea in 27 cases were graded as grade 3 and in 10 patients were graded as grade 4 . Tracheal resection with end-to-end anastomosis was performed in 16 patients, endotracheal laser treatment was performed in 11 patients, and in 10 patients t-tube stent was used. The laser treatment was performed by microlaryngotracheoscopy using jet ventilation intubation without using of the rigid bronchoscope. In the 10 cases of $\mathrm{T}$-tube placement dilatation was performed. The 10 patients with a grade 4 tracheal stenosis had tracheostomy performed previously. The VE and MPR were used to define the length of stenosis and the length of planned segment resection of the trachea.

The analysis between surgical findings versus FT and CT findings concerning stenosis grading, length, distance from vocal cord and the length of planned treatment segment of the trachea was performed. The grade of stenosis was correctly assessed by FT in 35/37 patients (94.6\%). CT measurements (MPR, VE) correctly estimated 36/37 (97.3\%) patients. The postoperative assessment of the length of stenosis was correctly assessed by FT in 34/37 patients (92\%). CT measurements (MPR, VE) correctly estimated 35/37 (94.6\%) patients. The postoperative measurement of the length of resected segment was considered accurate in $35 / 37$ (94.6\%) of the detected stenosis by CT and in 34/37 (92\%) of stenosis detected by FT. The postoperative assessment of the length from vocal cord was correctly assessed by FT in 33/37 patients (89\%). CT measurements (MPR, VE) correctly estimated 35/37 (94.6\%) patients. Complete correlation of stenosis grading, stenosis length, length from vocal cord, and length of planned resection segment of the trachea was noted between 33 of 37 patients with tracheal stenosis. Correlation between VE and intra-operative was noted in 35 of 37 patients with tracheal stenosis. Correlation between FT and VE was noted in 33 of 37 patients with tracheal stenosis. All other patients had a difference of only one score between VE, FT and intra-operative findings.

Grading of tracheal stenosis with FT, VE and MPR compared with an evaluation based on postoperative findings in the group of 37 patients with tracheal stenosis was demonstrated in Table 1. Virtual endoscopy was very useful in evaluating the lumen of the trachea beyond the impermeable segment in order to assess airways behind the lesion. There were no statistically significant differences between the intra-operative findings and FT or VE. 
Table 1 Grading of tracheal stenosis and correlation of postoperative grading with FT, VE, axial, coronal and sagittal reformatted CT of 37 patients with tracheal stenosis

\begin{tabular}{|c|c|c|c|c|c|}
\hline $\begin{array}{l}\text { Estimation } \\
\text { of stenosis } \\
\text { grading } \\
(N=37)\end{array}$ & $\begin{array}{l}\text { FT } \\
(\%)\end{array}$ & $\begin{array}{l}\mathrm{VE} \\
(\%)\end{array}$ & $\begin{array}{l}\text { Axial } \\
\text { CT }(\%)\end{array}$ & $\begin{array}{l}\text { Coronal } \\
\text { reformatted } \\
\text { CT }(\%)\end{array}$ & $\begin{array}{l}\text { Sagittal } \\
\text { reformatted } \\
\text { CT }(\%)\end{array}$ \\
\hline $\begin{array}{l}\text { Correctly } \\
\text { graded } \\
\text { stenosis }\end{array}$ & $\begin{array}{l}35 / 37 \\
\quad(94.6)\end{array}$ & $\begin{array}{l}36 / 37 \\
(97.3)\end{array}$ & $\begin{array}{l}35 / 37 \\
(94.6)\end{array}$ & $\begin{array}{l}35 / 37 \\
(94.6)\end{array}$ & $\begin{array}{l}35 / 37 \\
(94.6)\end{array}$ \\
\hline
\end{tabular}

Table 2 Analysis of the length of planned resection segment of trachea and correlation of postoperative findings with FT, coronal and sagittal reformatted CT of 37 patients with tracheal stenosis

\begin{tabular}{llll}
\hline $\begin{array}{l}\text { Estimation of length of } \\
\text { planned resection segment } \\
(N=37)\end{array}$ & $\begin{array}{l}\text { FT } \\
(\%)\end{array}$ & $\begin{array}{l}\text { Coronal } \\
\text { reformatted } \\
\text { CT }(\%)\end{array}$ & $\begin{array}{l}\text { Sagittal } \\
\text { reformatted } \\
\text { CT }(\%)\end{array}$ \\
\hline $\begin{array}{l}\text { Length of planned } \\
\text { resection segment }\end{array}$ & $32 / 37(86.5)$ & $35 / 37(94.6)$ & $35 / 37(94.6)$ \\
\hline
\end{tabular}

Analysis of the length of planned resection segment of trachea and correlation of postoperative findings with FT, coronal and sagittal reformatted CT was demonstrated in Table 2. The coronal and sagittal reformatted CT are very useful in planning of the stenosis resection and the accuracy is $94 \%$.

The analysis between FT versus CT findings concerning stenosis grading, length, distance from vocal cord, and the length of planned treatment segment of the trachea are presented in Table 3. Sensitivity was $94-97 \%$ and specificity was $100 \%$ in comparison with intra-operative findings. Measurements based on MPR reconstructions showed
86-89\% sensitivity and $100 \%$ specificity in comparison with FT. The PPV for stenosis measurements which were performed based on MPR reformations was $91-100 \%$.

\section{Discussion}

Conventional endoscopy, an invasive procedure for most surgeons, remains the gold standard for the identification and characterization of airway lesions of any size [18]. Endoscopy, however, may fail to provide sufficient information when the lesion is completely obstructing trachea, thus making it impossible to assess distal segments. Also, conventional endoscopy in some patients is impossible to perform or to finish, because of the presence of severe bleeding following a biopsy attempt, or because of some concomitant conditions (advanced age, tracheomalacia, etc.) [18]. In addition, it is an uncomfortable, poorly tolerated procedure that requires local sedation $[7,8,12,13]$ and is associated with a $0.8 \%$ morbidity $[7,15,18]$.

The present study demonstrated the high diagnostic accuracy, sensitivity, and specificity of virtual endoscopy. Finkelstein et al. [6] found that VE had a sensitivity of $100 \%$ for the detection of obstructive lesions. Shitrit et al. [21] found the evaluation of bronchial stenosis by VE to be highly correlated not only with FT, as reported previously, but also with pulmonary function test. Hoppe et al. [11] demonstrated that virtual endoscopy images, axial CT images alone, and multiplanar reformatted images were found to be highly accurate (VE images, 98\%; axial CT slices and reformatted coronal images, 96\%; reformatted sagittal images, 96.5\%) in the depiction of tracheobronchial stenosis. Taha et al. [23] evaluated the usefulness and accuracy of spiral CT in detection and assessment of post-

Table 3 Analysis and correlation between FT and VE, axial CT, coronal and sagittal reformatted CT of 37 patients with tracheal stenosis

\begin{tabular}{|c|c|c|c|c|c|c|c|c|c|c|}
\hline Examined features & $\mathrm{TP}$ & $\mathrm{TN}$ & FP & FN & $\begin{array}{l}\text { SENS } \\
(\%)\end{array}$ & $\begin{array}{l}\text { SPEC } \\
(\%)\end{array}$ & $\begin{array}{l}\text { PPV } \\
(\%)\end{array}$ & $\begin{array}{l}\text { NPV } \\
(\%)\end{array}$ & $\begin{array}{l}\text { ACC } \\
(\%)\end{array}$ & $\begin{array}{l}\text { Statistical } \\
\text { significance } \\
\text { (McNemara test) }\end{array}$ \\
\hline \multicolumn{11}{|l|}{ FT/VE } \\
\hline Stenosis grading & 33 & 2 & 0 & 2 & 94 & 100 & 100 & 50 & 95 & $\chi^{2}=0.5 ; p=0.48$ \\
\hline Stenosis length & 33 & 3 & 0 & 1 & 97 & 100 & 100 & 75 & 97 & $\chi^{2}=0 ; p=1$ \\
\hline Length from vocal cord & 32 & 1 & 3 & 1 & 97 & 25 & 91 & 50 & 89 & $\chi^{2}=0.25 ; p=0.62$ \\
\hline Length of planned resection segment of the trachea & 32 & 0 & 3 & 2 & 94 & 0 & 91 & 0 & 86 & $\chi^{2}=0 ; p=1$ \\
\hline \multicolumn{11}{|l|}{ FT } \\
\hline Axial CT & 31 & 2 & 0 & 4 & 89 & 100 & 100 & 33 & 89 & $\chi^{2}=2.25 ; p=0.13$ \\
\hline Coronal reformatted $\mathrm{CT}$ & 30 & 1 & 1 & 5 & 86 & 50 & 97 & 17 & 84 & $\chi^{2}=1.5 ; p=0.22$ \\
\hline Sagittal reformatted CT & 30 & 2 & 0 & 5 & 86 & 100 & 100 & 29 & 86 & $\chi^{2}=3.2 ; p=0.07$ \\
\hline
\end{tabular}

Examined patients $(N=37)$

$F T$ flexible tracheobronchoscopy, $V E$ virtual endotracheoscopy, $T P$ true positive, $T N$ true negative, $F P$ false positive, $F N$ false negative, SENS sensitivity, $S P E C$ specificity, $P P V$ positive predictive value, $N P V$ negative predictive value, $A C C$ accuracy 
intubation tracheal stenosis. The sensitivity and specificity of both CT and tracheobronchoscopy in the detection of subglottic stenosis was $100 \%$.

Virtual endoscopy is not operator dependent [19], and reports have demonstrated a $63-100 \%$ sensitivity and a 61-99\% specificity for this method for the identification of central stenosis $[4,7,11,12]$. Nevertheless, virtual examinations to date are unable to replace conventional imaging because of several intrinsic limitations. These include the inability to perform biopsies for histological assessment [5, $7,12]$ and to provide distinction in color or texture between normal mucosa and pathological tissue [4, 14, 18].

A major advantage of VE when compared with FT is its noninvasiveness; the method can be used in patients who are not able to undergo or whose parents refuse conventional endoscopy. Furthermore, VE can depict passage through high-grade stenoses, which enables evaluation of the poststenotic airway segments [9]. Finally, VE can be complementary to FT in the interventional setting, including stent implantation or tracheotomy. Disadvantages of VE include the inability to perform biopsies and therapeutic maneuvers and that color representation of mucosal surface in VT is artificial.

In the present study, negative predictive value (NPV) was low there were five false-negative findings with MPR reconstruction and only two with VE (patients with tracheobronchomalacia and normal findings at multidetector CT with VT). Similar results were reported by Heyer et al. [9]. Heyer et al. [9] reported that NPV was low; there were five false-negative findings with multidetector CT with VE (patients with tracheobronchomalacia and normal findings at multidetector CT with VE). This can be explained by the fact that tracheomalacia and bronchomalacia do not account for fixed narrowing and therefore can be reliably diagnosed only with functional studies. On the other hand Sun et al. [22] had $11.1 \%$ false results and $92 \%$ stenosis detection rate.

The present study demonstrated that VE which when integrated with MPR images, provided data for the detailed information of tracheal stenosis like grading, stenosis length, length from vocal cord and length of planned resection segment of the trachea before treatment. Thus information can be helpful for the planned treatment. Taha et al. [23] evaluated the usefulness and accuracy of spiral $\mathrm{CT}$ in detection and assessment of post-intubation tracheal stenosis. The results were compared with the intra-operative findings. They reported that detection rate for tracheal stenotic lesions was $94 \%$ by CT and $88 \%$ by rigid bronchoscopy. The preoperative assessment of the length of stenosis was accurate in $87 \%$ of the stenotic segments detected by CT and in $73 \%$ of the segments detected by bronchoscopy. The length of stenosis as assessed intraoperatively significantly correlated with the data obtained from CT examination $(r=0.98, p<0.001)$ and rigid tracheobronchoscopy $(r=0.94, p<0.001)$. The grade of stenosis was correctly assessed by bronchoscopy in $86 \%$ of patients.

The problem is when the stenosis can lead to progressive, often debilitating airflow obstruction that may be difficult to clinically differentiate from other causes of airflow limitation [20]. The correct diagnosis is important because, in most cases, the stenosis can be successfully treated with laser or by dilation and stent placement [10]. FT is the current standard for diagnosis of anastomotic complications, including stenosis [2]. However, it is invasive and may not be well tolerated. FT also provides only limited information on the length of the stenosis or the patency of the distal airways, which are important factors in planning treatment [8]. Nevertheless, VE may provide important diagnostic and potentially therapeutic information before FT is undertaken. It can also be used to evaluate patients with known tracheobronchial stenosis after treatment and may thereby reduce the frequency of repeated invasive FT performed for that purpose.

The 3D reconstruction software allows the user to navigate through the tracheobronchial tree [18] with same endoluminal perspective as conventional endoscopy [19, 24]. The virtual endoscopy images are displayed on the monitor alongside the multiplanar CT axial, sagittal and coronal oblique reference sections, on which the position and direction of the "virtual" bronchoscope is marked with a cursor. This makes it possible to explore the tracheobronchial tree from the inside by moving the cursor on the reference CT sections or by "navigating" the airway with a kind of "virtual airplane", with which direction and speed are controlled manually [17]. Some authors claimed that the information provided by spiral CT scan with MPR and VE may be considered as a substitute to direct endoscopic examination $[1,16]$.

This policy can minimize patient morbidity and spare them an extra anesthetic for evaluation. VE in multiview mode may also substitute conventional bronchoscopy in the follow-up of patients after endobronchial procedures in cases where the monitoring of changes in stenosis degree is important and successive bronchoscopy are unpleasant for the patient $[1,11]$. In the view of these findings, MPR and VE can be proposed as preliminary investigation to accurately characterize stenotic lesions, shorten conventional endoscopy time, and plan the most appropriate therapy.

In conclusion, the results of our study indicate that VE is an excellent, consistent and objective technique. VE with MPR is very useful in diagnostic evaluation and treatment planning in patients with tracheal stenosis.

\section{Conflict of interest None.}


Open Access This article is distributed under the terms of the Creative Commons Attribution Noncommercial License which permits any noncommercial use, distribution, and reproduction in any medium, provided the original author(s) and source are credited.

\section{References}

1. Bauer TL, Steiner KV (2007) Virtual bronchoscopy: clinical applications and limitations. Surg Oncol Clin N Am 16: 323-328

2. Colt HG, Janssen JP, Dumon JF et al (1992) Endoscopic management of bronchial stenosis after double lung transplantation. Chest 10291:10-16

3. Esclamado R, Cummings C (1993) Management of the impaired airway in adults. Cummings C (ed) Otolaryngology head and neck surgery, 2nd edn. Mosby year Book Inc, New York, pp 1981-2019

4. Ferretti GR, Thony F, Bosson JL et al (2000) Benign abnormalities and carcinoid tumors of central airways. AJR Am J Roentgenol 174:1307-1313

5. Ferretti GR, Kocier M, Calaque O et al (2003) Follow-up after stent insertion in the tracheobronchial tree: role of helical computed tomography in comparison with fiberoptic bronchoscopy. Eur Radiol 13:1172-1178

6. Finkelstein SE, Summers RM, Nguyen DM, Stewart JH, Tretler JA, Schrump DS (2002) Virtual bronchoscopy for evaluation of malignant tumors of the thorax. J Thorac Cardiovasc Surg 123(5): 967-972

7. Finkelstein SE, Schrump DS, Nguyen DM et al (2003) Comparative evaluation of super high-resolution CT scan and virtual bronchoscopy for the detection of tracheobronchial malignancies. Chest 124:1834-1840

8. Fullerton DA, Campbell DN (1996) Airway problems in lung transplantation. Semin Respir Crit Care Med 17:187-196

9. Heyer CM, Nuesslein TG, Jung D et al (2007) Tracheobronchial anomalies and stenoses: detection with low-dose multidetector CT with virtual tracheobronchoscopy_comparison with flexible tracheobronchoscopy. Radiology 242:542-549

10. Higgins R, McNeil K, Dennis C et al (1994) Airway stenosis after lung transplantation: management with expanding metal stents. J Heart Lung Transplant 13:774-778

11. Hoppe H, Walder B, Sonnenschein M, Vock P, Dinkel HP (2002) Multidetector CT virtual bronchoscopy to grade tracheobronchial stenosis. AJR Am J Roentgenol 178:1195-1200
12. Hoppe H, Dinkel HP, Walder B et al (2004) Grading airway stenosis down to the segmental level using virtual bronchoscopy. Chest 125:704-711

13. Kagadis GC, Patrinou V, Kalogeropoulou CP et al (2001) Virtual endoscopy in the diagnosis of an adult double tracheal bronchi case. Eur J Radiol 40:50-53

14. Kauczor HU, Wolcke B, Fischer B et al (1996) Three-dimensional helical CT of the tracheobronchial tree: evaluation of imaging protocol and assessment of suspected stenoses with bronchoscopic correlation. AJR Am J Roentgenol 167:419-424

15. Liewald F, Lang G, Fleiter T et al (1998) Comparison of virtual and fiberoptic bronchoscopy. Thorac Cardiovasc Surg 46:361-364

16. Maniatis PN, Triantopoulou CC, Tsalafoutas IA et al (2006) Virtual bronchoscopy versus thin section computed tomography in evaluation of moderate and low grade stenoses: receiver operating characteristic curve analysis. Acta Radiol 47:48-57

17. Naidich DP, Funt S, Ettenger NA, Arranda C (1990) Hemoptysis: bronchoscopic correlations in 58 cases. Radiology 177:357-362

18. Polverosi R, Vigo M, Baron S, Rossi G (2001) Valutazione delle lesioni tracheobronchiali con TC spirale. Confronto traendoscopia virtuale e broncoscopia. Radiol Med 102:313-319

19. Rapp-Bernhardt U, Welte T, Budinger M, Bernhardt TM (1998) Comparison of three-dimensional virtual endoscopy with bronchoscopy in patients with oesophageal carcinoma infiltrating the tracheobronchial tree. Br J Radiol 71:1271-1278

20. Ross DJ, Belman MJ, Mohsenifar Z et al (1994) Obstructive flow-volume loop contours after single lung transplantation. J Heart Lung Transplant 13:508-513

21. Shitrit D, Valdsislav P, Grubstein A et al (2005) Accuracy of virtual bronchoscopy for grading tracheobronchial stenosis: correlation with pulmonary function test and fiberoptic bronchoscopy. Chest 128:3545-3550

22. Sun M, Armin E, Boiselle PM (2007) MDCT of the central airways: comparison with bronchoscopy in the evaluation of complications of endotracheal and tracheostomy tubes. J Thorac Imaging 22:136-142

23. Taha MS, Mostafa BE, Fahmy M, Ghaffar MK, Ghany EA (2009) Spiral CT virtual bronchoscopy with multiplanar reformatting in the evaluation of post-intubation tracheal stenosis: comparison between endoscopic, radiological and surgical findings. Eur Arch Otorhinolaryngol 266:863-866

24. Vining DJ, Liu K, Choplin RH, Haponik EF (1996) virtual bronchoscopy. relationships of virtual reality endobronchial simulations to actual bronchoscopic findings. Chest 109:549-553

25. Wain JC (2003) Postintubation tracheal stenosis. Chest Surg Clin N Am 13:231-235 